\title{
Radiation-Pressure Effects in Cold-Atom Absorption Spectroscopy and Electromagnetically Induced Transparency
}

\author{
Suying Bai', Xiaoxuan Han1, Yunchun Jiao', Liping Hao', Jianming Zhao1,2*, Suotang Jia",2 \\ ${ }^{1}$ State Key Laboratory of Quantum Optics and Quantum Optics Devices, Institute of Laser Spectroscopy, Shanxi University, \\ Taiyuan, China \\ ${ }^{2}$ Collaborative Innovation Center of Extreme Optics, Shanxi University, Taiyuan, China \\ Email: *zhaojm@sxu.edu.cn
}

How to cite this paper: Bai, S.Y., Han, X.X., Jiao, Y.C., Hao, L.P., Zhao, J.M. and Jia, S.T. (2017) Radiation-Pressure Effects in Cold-Atom Absorption Spectroscopy and Electromagnetically Induced Transparency. Journal of Modern Physics, 8, 1884-1893. https://doi.org/10.4236/jmp.2017.811111

Received: October 4, 2017

Accepted: October 24, 2017

Published: October 27, 2017

Copyright $\odot 2017$ by authors and Scientific Research Publishing Inc. This work is licensed under the Creative Commons Attribution International License (CC BY 4.0).

http://creativecommons.org/licenses/by/4.0/

\begin{abstract}
Radiation pressure due to the interaction between a probe light and cold atoms is investigated in a standard cesium magneto-optical trap. The radiation pressure alters the absorption spectroscopy of cold atoms, leading to line shapes and linewidths after resonant interaction that are different for positive and negative probe chirps. The difference is attributed to the radiation pressure of the probe laser, due to which atoms become accelerated at the resonance. The effect of the radiation pressure is also seen in electromagnetically induced transparency (EIT) involving an excited Rydberg level. The density matrix equation accounting for the radiation pressure is used to simulate the experiments. The simulations agree well with the measurements both for absorption and EIT spectra. We find that the effect of the radiation pressure is reduced at low probe intensities, and can be neglected when the probe intensity is smaller than $I_{\text {sat }} / 2$.
\end{abstract}

\section{Keywords}

Radiation Pressure, Interaction, Rydberg EIT

\section{Introduction}

Radiation pressure results from the interaction between light and atoms has been predicated by Maxwell [1] and Bartoli [2]. Radiation pressure has been used to optically manipulate neutral atoms and has been widely investigated in the recent few decades. Absorption and emission of photons by an atom irradiated by a resonant or quasi-resonant laser light yield a variation of the 
atomic momentum. The mean radiative force is usually considered as consisting of two parts. The first part is radiation pressure, pointing in the direction of the phase gradient of the laser wave, which is used to manipulate the neutral atoms; the second one, related to the intensity gradient of the laser, is called dipole force, which is used to realize optical dipole traps.

The interactions between light and atoms can be absorption, reflection, or some of both (the common case), in which case each absorption of a resonant photon on an atomic transition with wavelength, $\lambda$, alters the momentum of the atom by $\hbar k=\hbar 2 \pi / \lambda$ ( $\hbar$ is the Planck constant) in the direction of laser light, whereas the direction of spontaneously emitted photon of the atoms is random, such that there is, on average, no momentum change from spontaneous emission. As a result, the atom receives a momentum kick $\hbar k$ in the direction of light propagation. Deceleration of atomic beams, stopping atoms, velocity reversal [3], laser cooling [4] [5] [6] [7] and Zeeman slowing [8] are interesting applications of radiation pressure. The technique of laser cooling and trapping is particular significance for observation of photonic Josephson effect and quantum phase transitions of light [9] [10] [11]. The radiation pressure also plays an important role in launching beams in atomic clocks [12], pushing beams in 2D MOTs [13] and in low-velocity intense source (LVIS) [14], as well as in parametric amplification of the mechanical motion [15] and levitation of nanometer sized objects [16] [17] [18] [19]. Applications of quantized photon recoil include atom interferometers [20]. Recently, an elastic wave in an elastic solid induced with light pressure has been directly measured [21], which clearly indicates the transfer of momentum due to light recoil from the interaction between the light with the surface of the elastic solid. These experiments benefit from radiation pressure. On other hand, the radiation pressure accelerates and Doppler-shifts atoms, resulting in broadened spectra. The broadening of the spectra limits atom-based measurements and applications, such as ultrahighresolution spectroscopy.

Here we investigate the absorption spectrum of cold atoms in a standard magneto-optical trap (MOT) considering the radiation pressure induced due to the interaction between a probe laser and cold atoms. The radiation pressure alters the shape of the absorption spectra, showing different effects for positive and negative probe chirps. The phenomenon is modeled using the density matrix equation, accounting for the Doppler effect induced by the radiation pressure. In experiments we apply one more laser beam that couples to a Rydberg level, resulting in a three-level atomic system, further verifying our model of radiation pressure.

\section{Experimental Setup}

The experiment is performed in a standard cesium MOT with temperature $~ 100$ $\mu \mathrm{K}$ and peak density $\sim 10^{10} \mathrm{~cm}^{-3}$, measured with the method of absorption image. The MOT size is approximately $500 \mu \mathrm{m}$. Figure 1 shows a schematic of the 
(a)

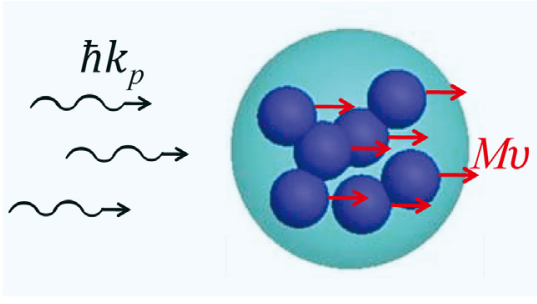

(c)

Trapping laser

(Coupling)

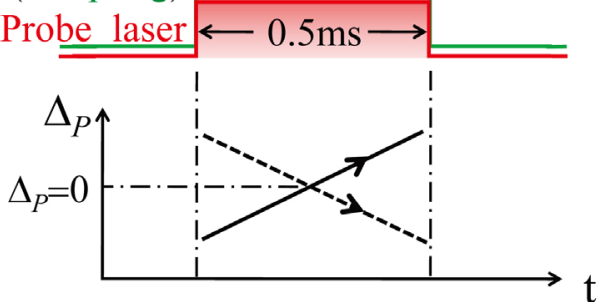

(b)

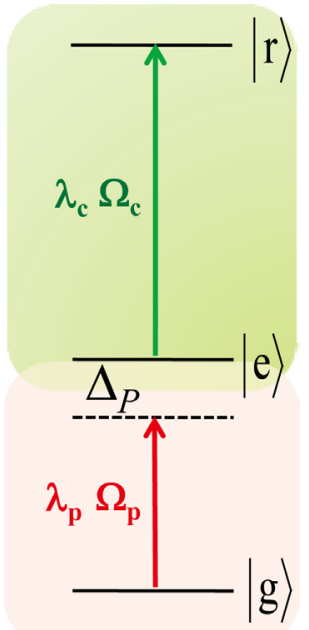

Figure 1. Schematic of the experiment (a) and relevant levels (b). The probe beam, $\lambda_{p}=852 \mathrm{~nm}$, Rabi frequency $\Omega_{p}=2 \pi \times 13 \mathrm{MHz}$, is tuned to near-resonance with the transition $\left|6 S_{1 / 2}, F=4\right\rangle(|g\rangle) \rightarrow\left|6 P_{3 / 2}, F^{\prime}=5\right\rangle(|e\rangle)$. (c) Timing: during the experiments, after switching off the MOT beam, both the probe and coupling lasers are switched on, and the probe frequency is positively (negatively) chirped over the resonance over a range of $\pm 25 \mathrm{MHz}$ in $0.5 \mathrm{~ms}$. The coupling laser is tuned into resonance with the Rydberg transition. The solid (dashed) black arrow shows the positive (negative) probe chirp. The absorption signal is detected as a function of the probe laser detuning, $\Delta_{p}$.

experiment (a) and the relevant energy levels (b). The probe laser, produced with a diode laser (DLpro, Toptica) with wavelength $\lambda_{p}=852 \mathrm{~nm}$ and linewidth 100 $\mathrm{kHz}$, is tuned to near resonance with the transition $|g\rangle \rightarrow|e\rangle$. The probe frequency is positively/negatively chirped through the $|g\rangle \rightarrow|e\rangle$ transition over a range $\pm 25 \mathrm{MHz}$, with 0.5 -ms chirp duration, see Figure 1 (c). The probe laser is detected with a photodiode detector as a function of the probe frequency detuning. The coupling laser, $\lambda_{c}=510 \mathrm{~nm}$, drives the Rydberg transition $|e\rangle \rightarrow|r\rangle$, completing the three-level cascade EIT system. The probe and coupling lasers are counter-propagated through the MOT center, corresponding Gaussian radius in the MOT are $\omega_{c}=120 \mu \mathrm{m}$ and $\omega_{p}=70 \mu \mathrm{m}$, respectively. The MOT magnetic field is always on during the experiments and the MOT-field gradient is $13 \mathrm{G} / \mathrm{cm}$, which results in a Zeeman broadening $<2 \pi \times 2 \mathrm{MHz}$, which is smaller than the natural linewidth of the $6 P_{3 / 2}$ level, $\Gamma_{e g}=2 \pi \times 5.2 \mathrm{MHz}$. The Rabi frequency, $\Omega$, of the transition, $|i\rangle \rightarrow|j\rangle$, is written as [22]

$$
\Omega=\frac{\mu_{i j}}{\hbar} \sqrt{\frac{2 P}{\pi \omega^{2} c \varepsilon_{0}}},
$$

where $\mu_{i j}$ is the transition dipole moment between two states $|i\rangle$ and $|j\rangle, P$ is the laser power coupling the transition and $\omega$ is the Gaussian waist of laser 
beam. $c$ is the speed of light and $\varepsilon_{0}$ is permittivity in vacuum.

\section{Absorption Spectra of the Cold Atoms Accounting for the Radiation Pressure}

In Figure 2 we show normalized spectra for a weak probe laser coupling the transition $|g\rangle \rightarrow|e\rangle$, with probe frequency chirped positively (negatively), as shown by the red and black curves. The spectra show the same line shape on the left side of the resonance point, $\Delta_{p}=0$, for positive and negative probe chirp, but show the different line shapes on the right side. Beyond the resonance, the line shape of the spectrum for positive-chirp probe shows a larger linewidth and more absorption, compared with the case of negative-chirp probe. The difference is attributed to the radiation pressure of the probe laser, due to which atoms become accelerated at the resonance. In the experiments, the atoms resonantly interact with the probe laser and pick up momentum in the direction of the probe beam when the probe laser frequency is resonant with the transition $|g\rangle \rightarrow|e\rangle$. This process accelerates the atoms and Doppler-shifts the transition $|g\rangle \rightarrow|e\rangle$. For the positive chirp, the increase of the probe-laser frequency compensates the Doppler shift in direction of the probe beam, which makes the atoms stay close to resonance somewhat longer, as the frequency sweep proceeds. This leads to more absorption on the blue-detuned side after the zero-detuning point, as shown by the absorption spectrum (red curve) in Figure 2. In contrast, for the negative chirp the change of the probe-laser frequency is opposite

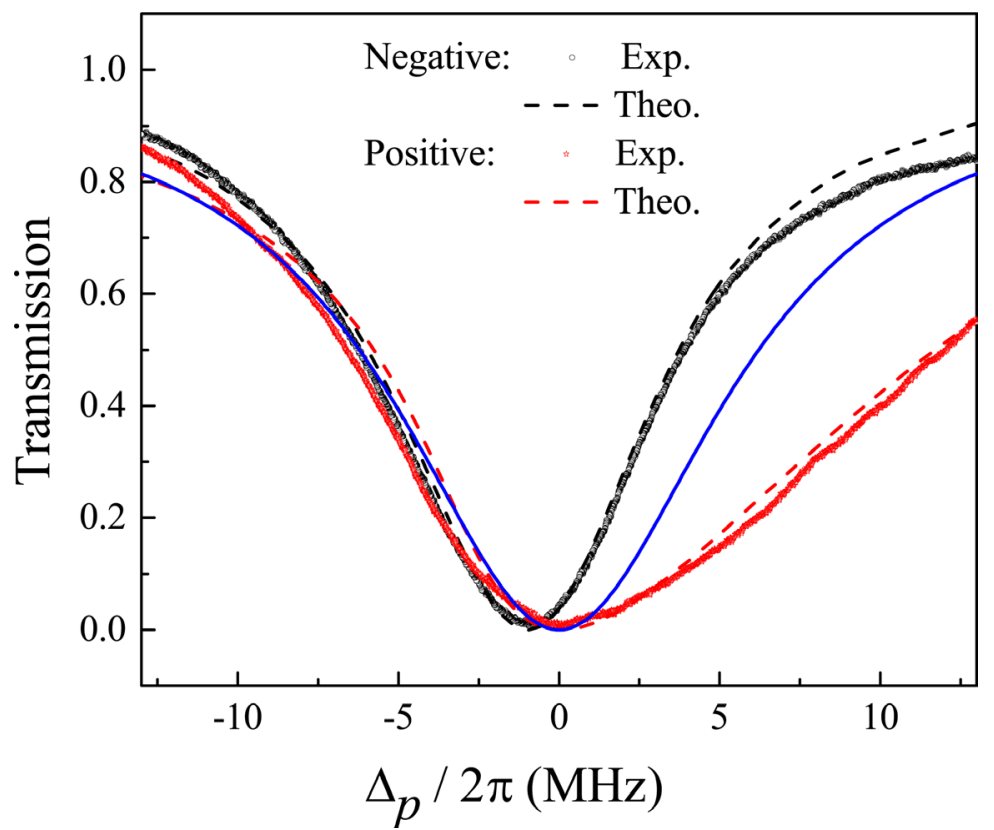

Figure 2. Normalized absorption spectra for measurements (symbols) and calculations (dashed lines), with positive (red stars) and negative (black circles) chirps of the probe laser over the $|g\rangle \rightarrow|e\rangle$ transition. The calculations are based on a two-level model with $\Omega_{p}=2 \pi \times 13 \mathrm{MHz}$, see text. The blue solid line represents the calculated absorption spectrum without accounting for the Doppler effect introduced by the radiation pressure. 
direction to the radiation-pressure-induced Doppler shift, leading to a faster decrease in absorption after passage through the zero-detuning point, as shown by the absorption spectrum (black curve).

For a full understanding of the measurements, we consider a probe laser (Rabi frequency $\Omega_{p}$ and detuning $\Delta_{p}$ ) interacting with an atom as shows in Figure 1 (b). The dynamics of the density matrix of the two-level system follows

$$
\begin{gathered}
\dot{\rho}_{g g}=\frac{i}{\hbar} \Omega_{p}\left(\rho_{g e}-\rho_{e g}\right)+\Gamma_{e g} \rho_{e e}, \\
\dot{\rho}_{g e}=\frac{i}{\hbar} \Omega_{p}\left(\rho_{g g}-\rho_{e e}\right)-\frac{2 i}{\hbar} \Delta_{p} \rho_{g e}-\frac{\gamma_{2}}{2} \rho_{g e}, \\
\dot{\rho}_{e g}=-\frac{i}{\hbar} \Omega_{p}\left(\rho_{g g}-\rho_{e e}\right)+\frac{2 i}{\hbar} \Delta_{p} \rho_{e g}-\frac{\gamma_{2}}{2} \rho_{e g}, \\
\dot{\rho}_{e e}=\frac{i}{\hbar} \Omega_{p} \rho_{e g}-\frac{i}{\hbar} \Omega_{p} \rho_{g e}-\Gamma_{e g} \rho_{e e}
\end{gathered}
$$

where $\Gamma_{e g}$ and $\gamma_{2}$ are the spontaneous decay and dephasing rates of the system, respectively, and $\hbar$ is the Planck constant.

As mentioned above, when the frequency of the probe laser is tuned into resonance with the transition $|g\rangle \rightarrow|e\rangle$, the atom absorbs the probe photons and picks up a momentum, $\hbar k_{p}, k_{p}=2 \pi / \lambda_{p}$, per photon. The atom is accelerated, and the corresponding velocity, $v(t)$, of the atom in the direction of probe beam is

$$
v(t)=\frac{\Gamma_{e g}}{M} \hbar k_{p} \int \rho_{e e}\left(t^{\prime}\right) \mathrm{d} t^{\prime}
$$

and the frequency detuning, $\Delta_{p}(t)$, the atom experiences is given by

$$
\Delta_{p}(t)=\Delta_{p 0} \pm \chi t-\frac{v(t)}{\lambda_{p}}
$$

here, $M$ is the mass of cesium. We do not account for the initial velocity of the atom in MOT because it has no significant effect. $\Delta_{p 0}$ is the start value of the probe-laser detuning, $\chi$ presents the probe-frequency chirp rate, and \pm represents the positive/negative sign of the frequency chirp.

We substitute $\Delta_{p}$ in Equations (2)-(5) with $\Delta_{p}(t)$ from Equation (7) and numerically solve the density matrix Equations (2)-(5). The photon scattering rates, $\Gamma_{e g} \rho_{e e}(t)$, lead to the calculated transmission of the probe beam as a function of the time-dependent probe detuning in Figure 2 (dashed lines). The calculations reproduce the spectra well for both positive and negative chirps and are consistent with the measurements well. Hence, it is seen that the radiation pressure yields Doppler shifts, induced by the interaction between probe laser and two-level atom. Different chirp polarities result in different line shapes of the probe spectra, obtained from cold-atom clouds. For comparison, we also plot the probe spectrum without accounting for the radiation pressure, shown with a blue solid line in Figure 2. 


\section{EIT Spectra Involving Rydberg State}

In order to further corroborate the explanation of absorption spectra in Sec. 3, we use electromagnetically induced transparency (EIT) involving a Rydberg state by applying a coupling laser, counter-propagating with the probe beam through the cesium MOT and driving the transition, $\left(6 P_{3 / 2}\right)|e\rangle \rightarrow\left(30 D_{5 / 2}\right)|r\rangle$, see Figure $1(b)$. EIT is a typical quantum interference effect in which a probe field experiences an increased transmission in an absorbing medium when a coupling field resonantly drives the up transition [23] [24]. EIT is widely investigated because of its special characteristics and enhanced refractive index, yielding slow light and storage of light pulse [25] [26]. In our experiments, we use levels $|g\rangle,|e\rangle$ and $|r\rangle$, constituting the three-level system. The probe laser is used to probe the EIT transparency, when the coupling laser is tuned to the Rydberg transition into $|r\rangle=\left|30 D_{5 / 2}\right\rangle$. Accounting for the Doppler shift due to the radiation pressure of the probe beam, the detuning of the coupling laser, $\Delta_{c}$, is written as

$$
\Delta_{c}(t)=\Delta_{c 0}+\frac{v(t)}{\lambda_{c}}
$$

The effect of the radiation pressure and resultant Doppler shifts make the EIT resonance condition different for the cases of positive and negative probe chirps. In Figure 3, we present the measured EIT spectra with Rabi frequency $\Omega_{c}=2 \pi \times 9.7 \mathrm{MHz}$ and $\Omega_{p}=2 \pi \times 13 \mathrm{MHz}$ with the probe laser for the positive

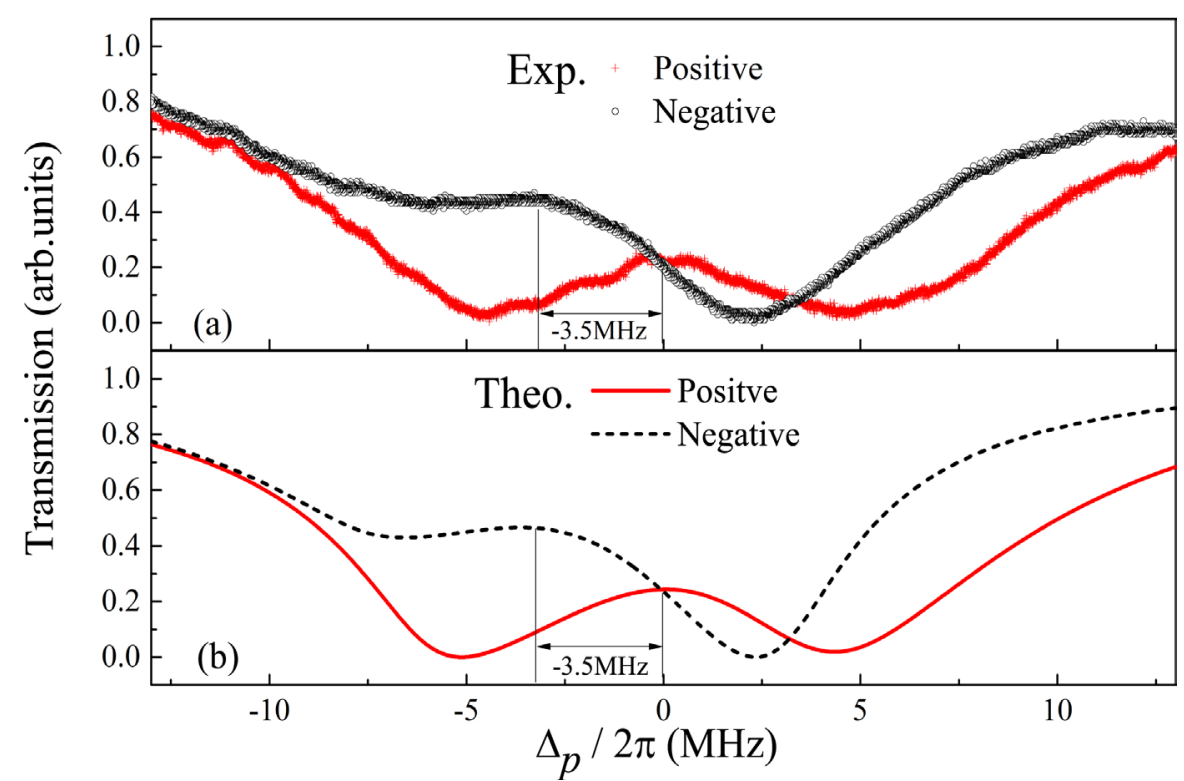

Figure 3. Measurements (a) and simulations (b) of EIT spectra for positively and negatively chirped probe lasers. The coupling laser drives the Rydberg transition, $30 \mathrm{D}_{5 / 2}$, and the corresponding Rabi frequencies $\Omega_{c}=2 \pi \times 9.7 \mathrm{MHz}$ and $\Omega_{p}=2 \pi \times 13 \mathrm{MHz}$. The Gaussian radius of the coupling and probe beams in the MOT are about $120 \mu \mathrm{m}$ and $70 \mu \mathrm{m}$, respectively. The EIT two-photon resonance for the case of positive chirp is used to define the 0 -detuning point. For negative probe chirp, the EIT peak appears at -3.5 MHz. 
and negative chirps. For simplicity, we calibrate the two-photon resonance condition (zero-detuning point) with the case of positive probe chirp, as demonstrated in Figure 3(a). In case of negative probe chirp, the EIT transparency peak appears at $3.5 \mathrm{MHz}$ on the red-detuned side, see Figure 3(a). The figure clearly shows that the EIT spectral profiles are different for positive and negative probe chirps. The difference of the EIT spectra in Figure 3 (a) is attributed to radiation pressure of the probe laser, as explained in Sec. 3.

For reproducing the EIT profiles, we numerically solve the density matrix equation expressed as

$$
\dot{\rho}=\frac{i}{\hbar}[H, \rho]+L
$$

where $H=H_{0}+H_{A L}$, and $H_{0}$ is the unperturbed Hamiltonian and $H_{A L}$ represents the interaction between light and atoms. $\rho$ is the density matrix of the three-level atom, see Figure 1(b), $L$ describes the decay and dephasing of the three-level system, for details see [27]. We take into account the radiation pressure and resultant Doppler shifts, Equations (6)-(8). The numerically calculated EIT spectra are presented in Figure $3(\mathrm{~b})$. The calculated EIT peak for negative chirp is $3.5 \mathrm{MHz}$ red-detuned compared with the positive case. This agrees well with the measurements in Figure 3(a). The simulation reproduces the experiments both in terms of the overall EIT spectral profiles and the EIT linewidths.

\section{Discussion}

The radiation pressure, induced due to the resonant interaction between the probe laser and atoms, results in the Doppler broadening and modification of the probe spectra profile on the blue-detuned side. This effect limits the precision of measurements and high-resolution spectroscopy based on cold atoms. For further investigation of how the radiation pressure affects the spectra, we change the probe laser intensity and perform similar measurements. Figure 4(a) shows the measured linewidths, $W$, of the probe spectra, defined as a full width at half maximum (FWHM), as a function of the probe intensities. The inset of Figure 4(a) shows four normalized absorption spectra for the indicated probe intensities. The measured linewidth for probe intensity $I_{p}=I_{\text {sat }}$ is $W_{\text {sat }}=10.1 \mathrm{MHz}$ for the experimental conditions here. Over the probe intensity range $(0.1-1) I_{\text {sab }}$ the change in linewidth is small, only about $15 \%$ of $W_{\text {sat }}$. In contrast, a large change is seen when the probe intensity exceeds $I_{\text {sat }}$. The linewidth more than doubles when the probe intensity reaches $10 I_{\text {sat }}$. It is noted that both radiation pressure and power (saturation) broadening affect the experimental spectra. The power broadening is homogeneous and symmetric. From Figure 4(a) inset, the power broadening can be estimated by the broadening on the left side of 0 -detuning point, while the radiation-pressure-induced broadening is given by the broadening on the right side. The latter is much larger than the former.

From Figure 4(a), the radiation-pressure-induced broadening can be neglect 

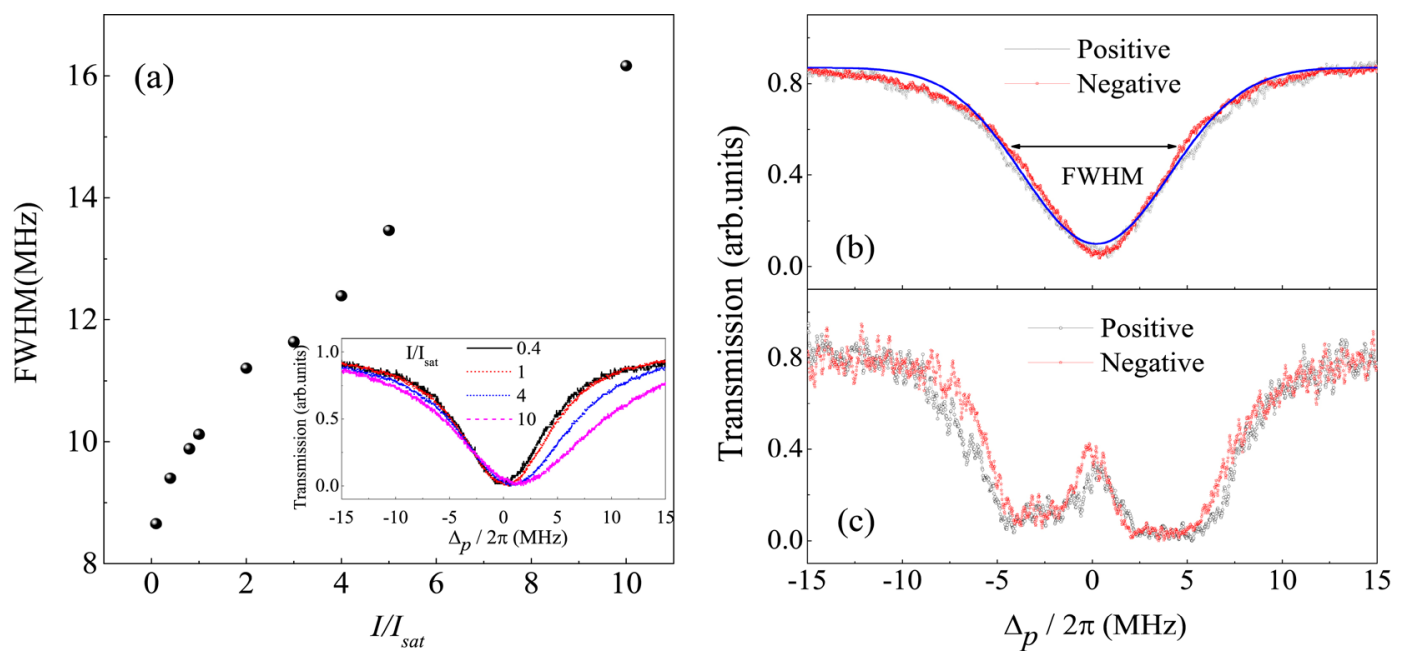

Figure 4. (a) Measured FWHM, $W$, of the probe absorption spectrum as a function of the probe intensity. The linewidth increases with the probe intensity; the effect of the radiation pressure can be ignored when probe intensity is smaller than $I_{\text {sat }} / 2$. Inset: four normalized absorption spectra with indicated probe intensities. Measurements of absorption (b) and EIT (c) spectra for positive (black circles) and negative (red stars) probe chirp. The probe laser intensity is $0.2 I_{\text {sat }}$, detected using an APD for getting high resolution and signal-to-noise spectra. The blue solid line in (b) is a Gaussian fit to the spectra. In the EIT spectra in (c), the coupling laser couples the Rydberg transition, $|r\rangle=\left|36 D_{5 / 2}\right\rangle$. In the case of low probe intensity, the spectra for positive and negative probe chirps overlap each other in both the absorption (b) and EIT spectra (c).

when the probe intensity is less than $I_{\text {sat }} / 2$. Figure 4(b) and Figure 4(c) present absorption (b) and EIT (c) spectra for positive and negative probe chirps at low probe intensity, $I_{p}=0.2 I_{\text {sat }}$. The coupling laser drives the Rydberg transition to $|r\rangle=\left|36 D_{5 / 2}\right\rangle$. Both the absorption in Figure $4(\mathrm{~b})$ and EIT transparency in Figure 4(c) show indistinguishable spectral profile for positive and negative probe chirps. This is in stark contrast to the high-intensity cases in Figure 2 and Figure 3. The EIT spectra in Figure 4(c) are slightly asymmetric due to a non-zero detuning of the coupling laser.

The low-intensity FWHM linewidth of the probe spectra is measured to be $2 \pi \times 8.6 \mathrm{MHz}$ (for $I_{p}=0.1 I_{\text {sat }}$ ). This is larger than the spontaneous decay rate of the level $|e\rangle, \Gamma_{e g}=2 \pi \times 5.2 \mathrm{MHz}$. The difference is mainly attributed to collision-induced broadening at large density of cold atoms, and MOT magnetic field induced Zeeman broadening due to MOT field on during experiments in this work.

\section{Conclusion}

In summary, we investigate the effect of the radiation pressure on absorption and EIT spectra obtained with cold atoms in a MOT. The probe spectra show different behavior for positive and negative probe frequency chirps, when the probe intensity is larger than $I_{\text {sat }}$. A positive frequency chirp compensates the Doppler shift in the direction of the probe beam, as the frequency sweep proceeds, leading to more absorption on the blue detuned side after passing the 
zero-detuning point. For the case of the negative chirp, the change of the probe-laser frequency is in opposite directions, leading to a faster decrease in absorption after resonance. The radiation pressure effect is verified further by applying a coupling laser, which couples the intermediate level to a Rydberg state. The theoretical model we have provided reproduces the spectra well both for the probe absorption spectra and the EIT spectra. The radiation pressure effect alters the spectral profiles, thereby limiting applicability to atom-based precision measurement. Using probe laser intensities smaller than $I_{\text {sat }} / 2$ or less probe-pulse duration, the radiation pressure effect can be effectively eliminated. The result obtained here serves as a guide how to reduce the radiation pressure effect.

\section{Acknowledgements}

We would like to thank Professor Georg Raithel (Ann Arbor) for stimulating discussions. The work was supported by the National Key R\&D Program of China (Grant No. 2017YFA0304203); National Natural Science Foundation of China (NSFC) (Grants No. 61475090, and No. 61675123); Changjiang Scholars and Innovative Research Team in University of Ministry of Education of China (Grant No. IRT13076); State Key Program of National Natural Science of China (Grant No. 11434007); Research Project Supported by Shanxi Scholarship Council of China (2014-009).

\section{References}

[1] Maxwell, J.C. (1873) A Treatise on Electricity and Magnetism. Clarendon Press, Oxford, Vol. 2.

[2] Bartoli, A. (1884) Il Nuovo Cimento, 15, 193-202. https://doi.org/10.1007/BF02737234

[3] Ertmer, W., Blatt, R., Hall, J.L. and Zhu, M. (1985) Physical Review Letters, 54, 996-999. https://doi.org/10.1103/PhysRevLett.54.996

[4] Chu, S. (1998) Reviews of Modern Physics, 70, 685-706. https://doi.org/10.1103/RevModPhys.70.685

[5] Cohen-Tannoudji, C.N. (1998) Reviews of Modern Physics, 70, 707-719. https://doi.org/10.1103/RevModPhys.70.707

[6] Phillips, W.D. (1998) Reviews of Modern Physics, 70, 721-741. https://doi.org/10.1103/RevModPhys.70.721

[7] Ashkin, A. (1978) Physical Review Letters, 40, 729-732. https://doi.org/10.1103/PhysRevLett.40.729

[8] Phillips, W.D. and Metcalf, H. (1982) Physical Review Letters, 48, 596-599. https://doi.org/10.1103/PhysRevLett.48.596

[9] Ji, A.C., Sun, Q., Xie, X.C. and Liu, W.M. (2009) Physical Review Letters, 102, Article ID: 023602. https://doi.org/10.1103/PhysRevLett.102.023602

[10] Ji, A.C., Xie, X.C. and Liu, W.M. (2007) Physical Review Letters, 99, Article ID: 183602. https://doi.org/10.1103/PhysRevLett.99.183602

[11] Zhang, X.F., Sun, Q., Wen, Y.C., Liu, W.M., Eggert, S. and Ji, A.C. (2013) Physical Review Letters, 110, Article ID: 090402. 
https://doi.org/10.1103/PhysRevLett.110.090402

[12] Kasevich, M.A., Riis, E., Chu, S. and DeVoe, R.G. (1989) Physical Review Letters, 63, 612-615. https://doi.org/10.1103/PhysRevLett.63.612

[13] Dieckmann, K., Spreeuw, R.J.C., Weidemüller, M. and Walraven, J.T.M. (1998) Physical Review A, 58, 3891-3895. https://doi.org/10.1103/PhysRevA.58.3891

[14] Lu, Z.T., Corwin, K.L., Renn, M.J., Anderson, M.H., Cornell, E.A. and Wieman, C.E. (1996) Physical Review Letters, 77, 3331-3334. https://doi.org/10.1103/PhysRevLett.77.3331

[15] Kippenberg, T.J., Rokhsari, H., Carmon, T., Scherer, A. and Vahala, K.J. (2005) Physical Review Letters, 95, Article ID: 033901. https://doi.org/10.1103/PhysRevLett.95.033901

[16] Singh, S., Bhattacharya, M., Dutta, O. and Meystre, P. (2008) Physical Review Letters, 101, Article ID: 263603. https://doi.org/10.1103/PhysRevLett.101.263603

[17] Millen, J., Fonseca, P.Z.G., Mavrogordatos, T., Monteiro, T.S. and Barker, P.F. (2015) Physical Review Letters, 114, Article ID: 123602. https://doi.org/10.1103/PhysRevLett.114.123602

[18] Barker P.F. and Shneider, M.N. (2010) Physical Review A, 81, Article ID: 023826. https://doi.org/10.1103/PhysRevA.81.023826

[19] Romero-Isart, O., Juan, M.L., Quidant, R. and Cirac, J.I. (2010) New Journal of Physics, 12, Article ID: 033015. https://doi.org/10.1088/1367-2630/12/3/033015

[20] Berman, P. (1996) Atom Interferometry. Academic Press, London.

[21] Požar, T. and Možina, J. (2013) Physical Review Letters, 111, Article ID: 185501. https://doi.org/10.1103/PhysRevLett.111.185501

[22] Zhang,H., Wang, L.M., Chen, J., Bao, S.X., Zhang, L.J., Zhao, J.M. and Jia, S.T. (2013) Physical Review A, 87, Article ID: 033835. https://doi.org/10.1103/PhysRevA.87.033835

[23] Boller, K.J., Imamoğlu, A. and Harris, S.E. (1991) Physical Review Letters, 66, 2593. https://doi.org/10.1103/PhysRevLett.66.2593

[24] Fleichhauer, M., Imamoglu, A. and Marangos, J.P. (2005) Reviews of Modern Physics, 77, 633-673. https://doi.org/10.1103/RevModPhys.77.633

[25] Lin, Y.W., Liao, W.T., Peters, T., Chou, H.C., Wang, J.S., Cho, H.W., Kuan, P.C. and Ite, A.Y. (2009) Physical Review Letters, 102, Article ID: 213601. https://doi.org/10.1103/PhysRevLett.102.213601

[26] Novikova, I., Walsworth, R.L. and Xiao, Y.H. (2012) Laser and Photonics Reviews, 6, 333-353. https://doi.org/10.1002/lpor.201100021

[27] Jiao, Y.C., Han, X.X., Yang, Z.W., Zhao, J.M. and Jia, S.T. (2016) Chinese Physics Letters, 33, Article ID: 123201. https://doi.org/10.1088/0256-307X/33/12/123201 